\title{
Euthanasia Method
}

National Cancer Institute

\section{Source}

National Cancer Institute. Euthanasia Method. NCI Thesaurus. Code C90336.

The mechanism by which a life is ended, usually in order to relieve pain and suffering. 